\title{
Evaluación de la Severidad de la Enfermedad Periodontal en Dientes Premolares Superiores en Comparación a los Dientes Premolares Inferiores en Pacientes Caninos
}

\author{
Assessment of the Severity of Periodontal Disease in Upper Premolars \\ Compared to the Lower Premolar Teeth in Canine Patients
}

\author{
Yanira Larraín N. ${ }^{1}$, Viviana Fernández P. ${ }^{1,2}$
}

\section{Resumen}

\begin{abstract}
El objetivo del presente estudio fue evaluar la severidad de la enfermedad periodontal en dientes premolares superiores en comparación a los premolares inferiores en pacientes caninos mayores de un año de edad, en la Clínica de Animales Menores de la Facultad de Medicina Veterinaria de la Universidad Nacional Mayor de San Marcos, durante el periodo octubre de 2012 a marzo de 2013. Se evaluaron todas las piezas dentarias de 54 canes mediante el Índice Veterinario Periodontal para establecer la presencia y severidad de enfermedad periodontal. El $81.5 \%$ de los perros presentaron algún grado de enfermedad periodontal y el $40 \%$ de los dientes estuvo afectados con algún grado de enfermedad periodontal. Asimismo, existe asociación entre la presencia de la enfermedad periodontal en dientes premolares y la arcada dentaria maxilar o mandibular, encontrándose que la enfermedad periodontal fue mayor en los dientes premolares superiores.
\end{abstract}

Palabras clave: enfermedad periodontal, perros, dientes premolares, arcada dentaria, índice veterinario periodontal

\section{AbSTRACT}

The aim of the present study was to assess the severity of periodontal disease in upper premolars compared to the lower premolars in canine patients older than one year old at the Small Animal Clinic of the Faculty of Veterinary Medicine of the Universidad Nacional Mayor de San Marcos, during the period October 2012 to March 2013. All teeth of 54 dogs were evaluated using the Veterinary Periodontal Index to stablish the presence

${ }^{1}$ Clínica de Animales Menores, Facultad de Medicina Veterinaria, Universidad Nacional Mayor de San Marcos, Lima, Perú

${ }^{2}$ E-mail:viviferpar@hotmail.com

Recibido: 26 de julio de 2016

Aceptado para publicación: 30 de diciembre de 2016 
and severity of periodontal disease. The results showed that $81.5 \%$ of dogs had some degree of periodontal disease and $40 \%$ of teeth were affected with some degree of periodontal disease. Moreover, there was an association between the presence of periodontal disease in tooth premolars and the maxillary or mandibular region, where periodontal disease was more frequent in the upper premolars.

Key words: periodontal disease, dogs, premolar teeth, dental arch, periodontal index veterinarian

\section{INTRODUCCIÓN}

Enfermedad periodontal es un término general que engloba un grupo de lesiones inflamatorias inducidas por la placa bacteriana que involucra al tejido de sostén del diente (Negro et al., 2003). La enfermedad periodontal es una de las patologías bucales más comunes en los perros, afectando al 85 90\% de los caninos mayores a 3 años (Gorrel, 1998), pero a su vez es una de las patologías orales que mejor se pueden prevenir (Hennet, 1995).

Esta enfermedad es progresiva y consta de dos fases: la gingivitis (reversible) y la periodontitis (irreversible, pero a menudo controlable). Es causada por la acumulación de placa en los dientes, la cual se mineraliza para formar cálculos que migran en el surco gingival causando la inflamación adicional, la pérdida de ligamento periodontal, la pérdida ósea y, en última instancia, la pérdida de dientes (Ford y Mazzaferro, 2007).

Una disminución en el número de dientes en perros puede ser causado por agenesia (mayormente en pequeñas razas braquiocefálicas), pero usualmente la mayor causa de pérdida dentaria es a consecuencia de enfermedad periodontal. Mayormente se reporta la pérdida del tercer molar inferior, los primeros premolares superiores e inferiores y los incisivos (Kyllar y Witter, 2005).

\section{Materiales y Métodos}

Se evaluaron 54 caninos mayores de un año de edad y de varias razas (excepto el Perro Sin Pelo del Perú) y de ambos sexos. Los canes eran procedentes de Lima Metropolitana y fueron llevados a la Clínica de Animales Menores de la Facultad de Medicina Veterinaria, Universidad Nacional Mayor de San Marcos, Lima, para la limpieza o control dental entre el 1 de octubre de 2012 y el 30 de marzo de 2013.

Los animales fueron manejados bajo anestesia general, empleando Diazepam 0.25 $\mathrm{mg} / \mathrm{kg}$ IV como premedicación y clorhidrato de ketamina $10 \mathrm{mg} / \mathrm{kg}$ IV para inducción y/o mantenidos con anestesia inhalatoria de isoflurano. Además, durante todo el procedimiento de destartarización y diagnóstico periodontal se administró fluidoterapia endovenosa.

El número de dientes presentes fue registrado. La fórmula dentaria estándar de la dentición permanente usada fue de $\mathrm{I} 3 / 3, \mathrm{C} 1 / 1$, P4/4, M2/3 (Page y Schroeder, 1982). Luego de la profilaxis dental, y empleando la sonda periodontal, se revisó la presencia de recesión gingival o hiperplasia gingival y la profundidad del surco gingival. Se registró, además, el sangrado al sondaje, el grado de movilidad dental y la afectación de furca en dientes multirradiculares, según Wiggs y Lobprise (1997). Este procedimiento se hizo por cada 
diente en seis lugares: posterior bucal, medio bucal, anterior bucal, posterior lingual/palatal, medio lingual/palatal y anterior lingual/palatal, con énfasis en los dientes premolares superiores e inferiores. Se otorgó un puntaje a cada pieza dentaria de acuerdo al Índice Veterinario Periodontal (IVP) que considera los siguientes valores (Wiggs y Lobprise, 1997):

- Sano (0). Profundidad al sondaje $<3 \mathrm{~mm}$. No hay sangrado ni signos de gingivitis.

- Gingivitis (1). Profundidad al sondaje $<3 \mathrm{~mm}$ con sangrado al sondaje, pero sin pérdida de unión.

- Periodontitis leve (2). Similar al grado 1 , pero con los primeros signos de periodontitis destructiva. Presencia de bolsa periodontal de $3-5 \mathrm{~mm}$, pérdida de unión de 0-2 mm, movilidad del diente de 0 o 1 y un grado de afección de furca de 0 o 1.

- Periodontitis moderada (3). Sangrado al sondaje, bolsa periodontal de 5-7 mm, pérdida de unión de $2-4 \mathrm{~mm}$, movilidad del diente de 1 o 2 y grado de afección de furca de 1 o 2 .

- Periodontitis severa (4). Sangrado al sondaje, bolsa periodontal $>7 \mathrm{~mm}$, pérdida de unión $>4 \mathrm{~mm}$, movilidad del diente de 2 o 3 y grado de afección de furca de 2 o 3 .

Cada pieza dentaria recibe un puntaje, y el cálculo del IVP de un paciente es la suma de los IVP de cada una de sus piezas dentarias entre el número de piezas examinadas. La interpretación del IVP fue la siguiente:
$<0.1=$ Normal o sano
$0.1-1=$ Gingivitis
$1.1-2=$ Periodontitis leve
$2.1-3=$ Periodontitis moderada
$3.1-4=$ Periodontitis severa

Se determinaron las frecuencias de las piezas dentarias y de pacientes con enfermedad periodontal. Se aplicó la prueba de Chi cuadrado para establecer la posible asociación entre la severidad de la enfermedad periodontal y su presencia en las arcadas dentarias superior e inferior, afección en dientes premolares de ambas arcadas, ausencia de dientes y demás tipo dentarios.

\section{Resultados}

Se evaluaron 2007 piezas dentarias, donde el $60 \%$ se encontraban sanas y el $40 \%$ presentaron algún grado de enfermedad periodontal. La presentación de la enfermedad periodontal en los dientes evaluados estuvo asociada a la arcada dentaria $(\mathrm{p}<0.05)$, siendo más susceptible la región maxilar con el $48.9 \%(n=464)$ de dientes enfermos en comparación con la región mandibular con el $32 \%(n=339)$ de dientes enfermos (Cuadro 1). Asimismo, se encontró una asociación estadística entre los dientes premolares según la arcada y la severidad de la enfermedad periodontal, donde el $59.6 \%$ de los dientes premolares superiores presentaron enfermedad periodontal en comparación con el $40.4 \%$ de los dientes premolares inferiores (Cuadro 2).

De las piezas dentarias premorales afectadas con gingivitis, el primer premolar inferior fue el más afectado (73.1\%), seguido del segundo, tercer y cuarto premolar inferior (61.1, 59.1 y $57.7 \%$, respectivamente). Con respecto a la periodontitis, los premolares superiores fueron más afectados que los inferiores; siendo el primer premolar superior afectado en mayor grado (67.4\%), seguido por el segundo, tercer y cuarto premolar (67.3, $66.7,60.6 \%$, respectivamente).

La ausencia dental fue mayor en los dientes premolares $(50.6 \%, 132 / 261)$, seguidos por los molares $(23.8 \%, 62 / 261)$, incisi$\operatorname{vos}(23.4 \%, 61 / 261)$ y caninos $(2.3 \%, 6 / 261)$. Así mismo, del total de dientes premolares ausentes, el primer premolar fue quien presentó un mayor porcentaje de ausencias, seguido por los segundos, terceros y cuartos premolares de ambas arcadas dentarias. 
Cuadro 1. Distribución porcentual de la presentación de enfermedad periodontal en la arcada dentaria superior e inferior en 54 pacientes caninos mayores de un año de edad de la Clínica de Animales Menores de la Facultad de Medicina Veterinaria, Universidad Nacional Mayor de San Marcos, Lima (octubre de 2012 a marzo de 2013)

\begin{tabular}{lcccccc}
\hline \multirow{2}{*}{$\begin{array}{l}\text { Región (arcada } \\
\text { dentaria) }\end{array}$} & \multicolumn{2}{c}{ Presente } & \multicolumn{2}{c}{ Ausente } & \multicolumn{2}{c}{ Total } \\
\cline { 2 - 7 } & $\mathrm{N} .{ }^{\circ 1}$ & $\%$ & $\mathrm{~N} .{ }^{\circ}$ & $\%$ & $\mathrm{~N}^{\circ}$ & $\%$ \\
\hline Maxilar & 464 & 48.9 & 485 & 51.1 & 949 & 47.3 \\
Mandibular & 339 & 32.0 & 719 & 68.0 & 1058 & 52.7 \\
\hline Total & 803 & 40.0 & 1204 & 60.0 & 2007 & 100.0 \\
\hline
\end{tabular}

${ }^{1}$ Número de dientes

Cuadro 2. Distribución porcentual de la severidad de la enfermedad periodontal en dientes premolares de las arcadas dentarias superior e inferior en 54 pacientes caninos mayores de un año de edad de la Clínica de Animales Menores de la Facultad de Medicina Veterinaria, Universidad Nacional Mayor de San Marcos, Lima (octubre de 2012 a marzo de 2013)

\begin{tabular}{lcccccc}
\hline \multirow{2}{*}{$\begin{array}{l}\text { Dientes } \\
\text { premolares }\end{array}$} & \multicolumn{2}{c}{ Gingivitis } & \multicolumn{2}{c}{ Periodontitis } & \multicolumn{2}{c}{ Total } \\
\cline { 2 - 7 } & $\mathrm{N} .^{\circ 1}$ & $\%$ & $\mathrm{~N} .^{\circ}$ & $\%$ & $\mathrm{~N} .^{\circ}$ & $\%$ \\
\hline Superiores & 42 & $21.9 \%$ & 150 & $78.1 \%$ & 192 & $59.6 \%$ \\
Inferiores & 50 & $38.5 \%$ & 80 & $61.5 \%$ & 130 & $40.4 \%$ \\
\hline Total & 92 & $28.6 \%$ & 230 & $71.4 \%$ & 322 & $100 \%$ \\
\hline
\end{tabular}

${ }^{1}$ Número de dientes

\section{Discusión}

La frecuencia de la enfermedad periodontal en los pacientes caninos del presente estudio (81.5\%) fue similar a los reportados por Hamp et al. (1984), Isogai et al. (1989), Kyllar y Witter (2005), Maetahara et al. (2010) y Rubiano et al. (2012). Además, el 40\% (803/2007) de los dientes examinados presentaron algún grado de enfermedad periodontal, según el Índice Veterinario de Enfermedad Periodontal (Wiggs y Lobprise, 1997), siendo este un valor similar al reporta- do por Paz et al. (2012), quien encontró $42.9 \%(728 / 1695)$ de dientes enfermos.

El elevado porcentaje de enfermedad periodontal puede deberse a la escasa o nula evaluación de la cavidad oral llevado a cabo por los profesionales médicos veterinarios, debido muchas veces al desconocimiento o subestimación de las repercusiones de esta patología sobre la salud oral y sistémica del paciente, las cuales han sido reportadas por varios autores (Debowes et al., 1996; Gorrel, 1998). Adicionalmente, Hoffman y Gaengler (1996) realizaron un estudio epidemiológico 
de esta enfermedad en perros Poodle en Alemania, hallando una prevalencia del $90 \%$ en canes menores de cuatro años.

En la evaluación de cada pieza dentaria se determinó que la arcada dentaria superior es más susceptible que la arcada dentaria inferior (60 vs. $40 \%$ ), resultado coincidente con los reportes de Killar y Witter (2005) y Maetahara et al. (2010), a diferencia de otros reportes donde no se observaron diferencias significativas entre arcadas (Hamp y Loe, 1973; Harvey et al., 1994; Hoffmann y Gaengler, 1996; Paz et al., 2012). La mayor frecuencia de enfermedad periodontal de la arcada superior pudo deberse a que las mayores superficies potenciales retentivas de placa se encuentran en esta arcada. Esto, por ejemplo, se puede encontrar en pacientes caninos de cráneo braquicefálico, ya que poseen dientes muy grandes para sus arcadas, resultando en un amontonamiento de dientes que originan problemas como rotación de premolares con ángulos de $90^{\circ}$ a $180^{\circ}$, así como retención de dientes deciduos y maloclusiones, lo cual permite que se creen superficies donde se acumulan restos de comida, predisponiendo la formación de placa y cálculos dentales (Colmery y Frost, 1986).

Gorrel (1998) indica que una de las razones por la mayor acumulación de placa y cálculos dentales en los premolares se deben a sus características anatómicas; pues las desembocaduras de los conductos de las glándulas salivales parótidas y cigomáticas se presentan entre el cuarto premolar y primer molar superiores, favoreciendo la deposición mineral constante para la formación rápida de sarro dental. Esto es consistente con lo encontrado en el presente trabajo donde la enfermedad periodontal fue mayor en los dientes premolares superiores $(59.6 \%)$ que en los inferiores (40.4\%). En forma similar, Harvey y Emily (1993) encontraron una mayor frecuencia y severidad de la enfermedad periodontal mayor en los dientes superiores, especialmente en los premolares.
Hennet y Orsini (1992) reportaron gingivitis más severas y mayor acúmulo de placa y cálculo en la región maxilar, especialmente en la cara bucal que en la cara lingual de los dientes (Eisenmenger y Zetner, 1985).

Page y Schroeder (1982) y Niemiec (2013) refieren que una de las causas más probables de pérdida dentaria es la disminución progresiva del hueso alveolar a consecuencia de la periodontitis. El presente estudio reveló que el 72.7\% (32/44) de los caninos presentaron enfermedad periodontal con ausencia de por lo menos una pieza dentaria $(\mathrm{p}<0.05)$. Asimismo, los premolares fueron las piezas dentarias de mayor ausencia $(50.6 \%, 132 / 261)$, seguido por los molares, incisivos y caninos. Por otro lado, la ausencia dental premolar más evidente fue la del primer premolar, que representó el $43.2 \%$ (57/132) del total de piezas premolares ausentes.

\section{Conclusiones}

- $\quad$ El $81.5 \%$ de los caninos (44/54) presentaron enfermedad periodontal.

- $\quad$ El 40\% de dientes evaluados (803/2007) presentaron algún grado de enfermedad periodontal.

- La arcada dentaria superior fue más susceptible que la arcada inferior a la presentación de la enfermedad periodontal.

- La enfermedad periodontal fue mayor en los dientes premolares superiores (59.6\%) que en los inferiores (40.4\%).

- Existe asociación entre la presentación de la enfermedad periodontal y la ausencia de dientes en los pacientes caninos.

\section{Literatura Citada}

1. Colmery B, Frost P. 1986. Periodontal disease: etiology and pathogenesis. Vet Clin North Am-Small 16: 817-833. doi: 10.1016/S0195-5616(86)50303-X 
2. Debowes LJ, Mosier D, Logan E, Harvey C, Lowry S, Richardson DC. 1996. Association of periodontal disease and histologic lesions in multiple organs from 45 dogs. J Vet Dent 13: 57-60.

3. Eisenmenger E, Zetner K. 1985. Periodontopatías. En: Odontología veterinaria. Barcelona, España: Ed Marzo 80. p 133-152.

4. Ford RB, Mazzaferro EM. 2007. Kirk e Bistner. Manual de procedimentos veterinários e tratamento emergencial. $8^{\circ}$ ed. São Paulo: Ed Roca. 760 p.

5. Gorrel C. 1998. Periodontal disease and diet in domestic dogs. J Nutr 128: 2712S2714S.

6. Hamp S, Loe H. 1973. Long term effects of chlorhexidine on developing gingivitis in the beagle dog. J Periodontal Res 8: 63-70.

7. Hamp S, Olsson S, Farso-Madsen K, Viklands P, Fornell J. 1984. A macroscopic and radiologic investigation of dental diseases of the dog. Vet Radiol Ultradound 25: 86-92. doi: 10.1111/j.17408261.1984.tb01916.x

8. Harvey CE, Emily P. 1993. Occlusion, occlusive abnormalities and orthodontic treatment. In: Ladig D (Ed). Small animal dentistry. St. Louis, MO: Mosby-Year Book. p 266-273.

9. Harvey C, Shofer F, Laster L. 1994. Association of age and body weight periodontal disease in North American dogs. J Vet Dent 11: 94-105.

10. Orsini P, Hennet P. 1992. Anatomy of the mouth and teeth of the cat. Vet Clin North Am-Small 22: 1265-1277. doi: 10.1016/S0195-5616(92)50126-7

11. Hennet P. 1995. Periodontal disease and oral microbiology. In: Crossley D, PenmanS (eds). Manual of small animal dentistry. $2^{\text {nd }}$ ed. UK: British Small Animal Veterinary Association. p 105-113.

12. Hoffman T, Gaengler P. 1996. Epidemiology of periodontal disease in poodles. J Small Anim Pract 37: 309-316. doi: 10.1111/j.1748-5827.1996.tb02396.x
13. Isogai H, Isogai E, Okamoto H, Shirakawa H, Nakamura F, Matsumoto T, Watanabe T, et al. 1989. Epidemiological study on periodontal diseases and some other dental disorders in dogs. Jpn J Vet Sci 51: 1151-1162.

14. Kyllar M, Witter K. 2005. Prevalence of dental disorders in pet dogs. Vet MedCzech 50: 496-505.

15. Maetahara A, Fernández V, Chipayo I, Suárez F. 2010. Frecuencia y severidad de la enfermedad periodontal en pacientes caninos de una clínica de animales menores. Rev Inv Vet Perú 21: 68-72. doi: 10.15381/rivep.v21i1.309

16. Negro S, Carloni G, Hernández SZ, Rodríguez D. 2003. Bacterias subgingivales en pacientes caninos con enfermedad periodontal. Informe Previo. In Vet 5(1): 114.

17. Niemiec B. 2013. Veterinary periodontology. San Diego, USA: Wiley Blackwell. $368 \mathrm{p}$.

18. Page R, Schroeder $H .1982$. Periodontitis in man and other animals. A comparative review. Basel: Karger. $330 \mathrm{p}$.

19. Pavlica Z. 2002. Enfermedad periodontal y sus efectos a nivel sistémico en la población canina de riesgo. En: Simposio «Control clínico y nutricional de perros y gatos senior». Granada, España.

20. Paz R, Fernández V, Suárez F, Sato A. 2012. Asociación entre el biotipo cefálico y la severidad de la enfermedad periodontal en caninos. Rev Inv Vet Perú 23: 147-152. doi: 10.15381/ rivep.v23i2.894

21. Rubiano D, Rojas D, Almansa J, Villalobos M, Montoya D, Urquijo G 2012. Frecuencia de enfermedad periodontal y caries en caninos del Centro de Zoonosis de Bogotá. Rev Nac Odontol 8(15): 21-29.

22. Wiggs R, Lobprise H. 1997. Periodontology. In: Veterinary dentistry principles and practice. Philadelphia, USA: Ed. Lippincott-Raven. p 186-231. 